\title{
Prática mental na reabilitação de disfunções neuromotoras: uma revisão integrativa
}

\author{
Mental practice in the rehabilitation of neuromotor \\ dysfunctions: an integrative review
}

\section{Práctica mental en la rehabilitación de disfunciones neuromotoras: una revisión integrativa}

\author{
Danielle de Paula Aprigio Alves ${ }^{1}$, Juliana Bittencourt Marques ${ }^{2}$, Alair \\ Pedro Ribeiro de Souza e Silva ${ }^{3}$, Bruna Brandão Velasques ${ }^{4}$
}

\begin{abstract}
1.Fisioterapeuta, Mestre em Saúde Mental, Laboratório de Neurofisiologia e Neuropsicologia da Atenção, Instituto de Psiquiatria da Universidade Federal do Rio de Janeiro (IPUB/UFRJ). Rio de Janeiro-RJ, Brasil. https://orcid.org/0000-0001-5970-3497

2.Fisioterapeuta, Doutora em Saúde Mental, Universidade Veiga de Almeida, Cabo Frio-RJ, Brasil. https://orcid.org/0000-0003-1959-6345

3.Educador Fisico, Doutor em Controle Motor, Professor Titular do Departamento de Biociências (EEFD/ UFRJ), Escola de Educação Fisica. Coordenador do Laboratório de Integração Sensório-Motora, Instituto de Psiquiatria da Universidade Federal do Rio de Janeiro (IPUB/UFRJ), Rio de Janeiro-RJ, Brasil. https://orcid.org/0000-0001-8270-3663

4.Psicóloga, Doutora em Saúde Mental, Coordenadora Laboratório de Neurofisiologia e Neuropsicologia da Atenção, Instituto de Psiquiatria da Universidade Federal do Rio de Janeiro (IPUB/UFRJ). Rio de JaneiroRJ, Brasil. https://orcid.org/0000-0001-9363-8932
\end{abstract}

\section{Resumo}

Introdução. Nas últimas décadas diferentes ferramentas em neurorreabilitação estão surgindo em detrimento a reabilitação física de pacientes com doenças neuromotoras. Entre essas, a imagética motora através da prática mental, parece promover melhora da performance motora e da funcionalidade em sujeitos com distúrbios neurológicos (p.ex. Acidente Vascular Cerebral, Doença de Parkinson, Esclerose Múltipla etc.). Objetivo. O objetivo do estudo é sintetizar a literatura relevante sobre a aplicabilidade de protocolos de treinamento com prática mental em neurorreabilitação, a fim de facilitar a prática do fisioterapeuta. Método. Para a realização deste estudo foi conduzida uma revisão da literatura do tipo integrativa, utilizando como fonte artigos indexados pelas bases de dados: Pubmed/Medline, Cochrane, Pedro e Scielo. Resultados. Primeiro o conceito de imagética motora é apresentado à luz das evidências. Logo, foram discutidos os parâmetros de avaliação e estratégias para a aplicabilidade da técnica. Em seguida, são analisados os protocolos disponíveis para o treinamento com prática mental em pacientes com disfunções neuromotoras. Conclusão. As evidências apontam a favor do uso da prática mental nas disfunções neuromotoras, sendo os resultados superiores quando combinada com a reabilitação convencional. Devido à grande heterogeneidade na literatura científica disponível, alguns achados são contraditórios e não há consenso sobre os protocolos mais adequados.

Unitermos. Imagética Motora; Prática Mental; Reabilitação; Neurologia

\footnotetext{
Abstract

Introduction. In the last decades, different tools in neurorehabilitation have been at the expense of physical rehabilitation of patients with neuromotor diseases. Among these, motor imagery through mental practice, seems to promote improvement in motor performance and functionality in subjects with neurological disorders (eg Stroke, Parkinson's Disease, Multiple Sclerosis etc.). Objective. The objective of the study is to synthesize the relevant literature on the applicability of training protocols with MI in neuroreabilitation, in order to facilitate the practice of the physical therapist. Method. To carry out this study, an integrative literature review was conducted, using articles indexed by the databases: Pubmed/Medline, Cochrane,
} 
Pedro and Scielo as a source. Results. First, the concept of motor imagery is presented in the light of evidence. Then, the evaluation parameters and strategies for the applicability of the technique were discussed. Then, the protocols available for training with mental practice in patients with neuromotor disorders are analyzed. Conclusion. The evidence points in favor of the use of mental practicein neuromotor disorders, the results being superior when combining with conventional rehabilitation. Due to the great heterogeneity in the available scientific literature, some findings are contradictory and there is no consensus on the most appropriate protocols.

Keywords. Motor Imagery; Mental Practice; Rehabilitation; Neurology

\begin{abstract}
Resumen
Introducción. En las últimas décadas, diferentes herramientas en neurorrehabilitación han estado disponibles para la rehabilitación física de pacientes con enfermedades neuromotoras. Entre ellas, la imaginería motora a través de la práctica mental parece promover la mejora del rendimiento y la funcionalidad motora de sujetos con trastornos neurológicos ( $p$. ej. Accidente Cerebrovascular, Enfermedad de Parkinson, Esclerosis Múltiple, etc.). Objetivo. El objetivo de este estudio es sintetizar la literatura relevante sobre la aplicabilidad de protocolos de entrenamiento con imaginería motora en neurorrehabilitación, con el fin de facilitar la práctica del fisioterapeuta. Método. Para la realización de este estudio se realizó una revisión integradora de la literatura, utilizando como fuente artículos indexados en las bases de datos: Pubmed/Medline, Cochrane, Pedro y Scielo. Resultados. Primero, el concepto de imaginería motora se presenta a la luz de la evidencia. Luego, se discutieron los parámetros de evaluación y las estrategias para la aplicabilidad de la técnica. Seguido a esto, se analizan los protocolos disponibles para el entrenamiento con práctica mental en pacientes con trastornos neuromotores. Conclusiones. La evidencia apunta a favor del uso de la práctica mental en trastornos neuromotores, consiguiendo mejores resultados cuando se combina con la rehabilitación convencional. Debido a la gran heterogeneidad en la literatura científica disponible, algunos hallazgos son contradictorios y no existe consenso sobre los protocolos más adecuados.
\end{abstract}

Palabras clave. Imaginación Motora; Práctica Mental; Rehabilitación; Neurología

Trabalho realizado no Laboratório de Neurofisiologia e Neuropsicologia da Atenção, Instituto de Psiquiatria da Universidade Federal do Rio de Janeiro (IPUB/UFRJ), Rio de Janeiro-RJ, Brasil.

\title{
INTRODUÇÃO
}

O treinamento com Imagética Motora (IM) através da Prática Mental (PM) está cada vez mais sendo recomendado em neurorreabilitação. Tem sido pensado como rota alternativa, uma porta de entrada ao sistema motor ${ }^{1}$. Diferentes protocolos de intervenção com PM têm sido utilizados em estudos envolvendo a reabilitação de disfunções neuromotoras. Em várias condições neurológicas a PM demonstrou ter efeitos benéficos, como complemento a Prática Física (PF). Resultados promissores são 
apresentados na recuperação após doenças cerebrovasculares, nas variáveis funcionalidade e mobilidade por exemplo ${ }^{1-3}$; nos transtornos do movimento ${ }^{4,5}$, em aspectos do equilíbrio e marcha; nas doenças desmielinizantes ${ }^{6}$; e, na lesão medular7. No entanto, o conteúdo dos protocolos de intervenção são diversos e raramente discutidos ou questionados.

A IM compreende, "imaginar-se realizando 0 movimento hábil sem realmente fazer o movimento". O sujeito pode imaginar o movimento na perspectiva de primeira ou de terceira pessoa ${ }^{8}$. As imagens podem ser experimentadas, envolvendo uma experiência cinestésica da ação (representação interna) - perspectiva de primeira pessoa; ou através da percepção visual do movimento imaginado (representação externa) - perspectiva de terceira pessoa $^{1}$. A PM descreve uma forma de treinamento na qual a representação interna do movimento é ativada e a execução desse é mentalmente simulada, sem que haja a excursão real do movimento, e isso acontece dentro de um contexto específico ${ }^{1}$.

Evidências convergentes indicam que as imagens motoras compartilham os mesmos substratos neurais envolvidos na programação, preparação e execução do movimento 9 . Estudos com Mapeamento Cerebral, Tomografia por Emissão de Pósitrons (PET) ${ }^{10}$ e Ressonância Magnética Funcional (RMF) ${ }^{5}$, tem auxiliado na compreensão das funções psicofísicas e neurofisiológicas entre movimentos executados e imaginados, com indícios de que 
esses se baseiem em um mesmo processo ${ }^{9}$. Esses adventos, corroboram com a noção de que as áreas corticais ativadas durante a imaginação motora são muito semelhantes as ativadas durante o planejamento e execução do movimento. Assim, parece lógico esperar que as mudanças centrais produzidas durante a IM devam também afetar 0 desempenho motor.

Apesar de já descritos os benefícios da PM, pouco se sabe sobre os parâmetros temporais e os elementos essenciais para o desempenho de um treinamento com IM em neurorreabilitação. Indicação e contraindicação, frequência, duração, intensidade, momento de implementação da terapia, são algumas questões que permanecem ${ }^{1,11}$. O uso de IM para indivíduos com disfunções neuromotoras, exige a definição de alguns desses parâmetros para se atingir a meta terapêutica e, consequentemente, ganhos funcionais. Os efeitos positivos do treinamento com IM de forma isolada não pôde ser alcançado ${ }^{12}$, mas a combinação de PM e PF produzem os melhores resultados ${ }^{2,13,14}$. A presente pesquisa tem como objetivo sintetizar a literatura relevante sobre a aplicabilidade de protocolos de treinamento com IM em neurorreabilitação, a fim de facilitar a prática do fisioterapeuta. De forma específica: (1) Conceituar os distintos termos empregados sobre imagética motora, identificando as estratégias para o uso da simulação mental; (2) Discutir as evidências quanto aos parâmetros de avaliação e aplicabilidade da técnica; e (3) Identificar os 
protocolos disponíveis para o uso da IM em pacientes com disfunções neuromotoras.

\section{MÉTODO}

Para a realização deste estudo foi conduzida uma revisão da literatura do tipo integrativa, utilizando como fonte artigos indexados nas bases dados: Pubmed/Medline, Cochrane, PEDro e Scielo publicados entre os anos de 1996 e 2020, nos idiomas inglês, espanhol e português. Como palavras-chave da pesquisa foram usados os seguintes descritores: Acidente Cerebrovascular, Traumatismo da Medula Espinhal, Doença de Parkinson, Esclerose Múltipla, Reabilitação, Neurologia, Imagética Motora e Prática Mental, para localizar estudos que abordassem os seguintes temas: avaliação da capacidade de imaginar, estratégias de IM, IM cinestésica e visual, PM e/ou simulação mental na reabilitação de doenças neuromotoras. Para esta revisão integrativa, seguiu-se as seguintes etapas: definição da questão norteadora; objetivo geral e específicos; coleta de dados baseado nos critérios de elegibilidade do estudo; categorização e avaliação dos estudos incluídos; análise dos resultados e síntese do conhecimento.

Foram considerados como critérios de inclusão:

- Quanto aos participantes: estudos em indivíduos com condições neurológicas clinicamente evidentes;

- Quanto a intervenção: estudos que utilizaram como técnica a PM/IM isolada ou combinada a prática física; 
- Quanto ao tipo de pesquisa: estudos de ensaio clínico randomizado; transversais; coorte; caso-controle; relato de caso e/ou revisão sistemática e metanálise.

Foram excluídos:

- Quanto aos participantes: estudos que abordaram outras condições que não fossem disfunções neuromotoras;

- Quanto a intervenção: estudos que não incluíram a PM/IM em seus protocolos de intervenção;

- Somente foram utilizados os artigos cujos textos completos puderam ser acessados.

\section{RESULTADOS}

Uma ampla quantidade de registros foi identificada a partir dos bancos de dados; após aplicação dos critérios de elegibilidade 26 foram selecionados para a realização desta revisão. Apenas 11 artigos de intervenção foram utilizados para análise da aplicabilidade de protocolos de PM em neurorreabilitação, e estes foram lidos na íntegra pelos avaliadores. A Tabela 1, descreve e resume as características dos estudos considerados potencialmente relevantes. Os dados extraídos para a caracterização dos estudos incluíram:

o primeiro autor e ano de publicação, população estudada (amostra), protocolo de intervenção, medidas, desfechos e resultados.

Na análise dos estudos expostos na Tabela 1, observouse quatro estudos referentes ao uso da IM no Acidente Vascular Cerebral12,14-16; quatro na Doença de 
Parkinson ${ }^{1,4,5,17}$; dois na Esclerose Múltipla ${ }^{18,19}$ e um na Lesão Medular $^{20}$.

As medidas utilizadas nos estudos foram diversas, envolvendo: testes cognitivos; questionários para análise da capacidade de imaginar; e o uso de instrumentos de avaliação funcional, estes em concordância com os domínios da Classificação Internacional de Funcionalidade (CIF). Em relação aos desfechos primários, alguns benefícios relatados após protocolo de intervenção envolveram, a funcionalidade de membro superior baseado nas atividades básicas de vida diárias ${ }^{12,16,20}$; mobilidade funcional - marcha ${ }^{1,5,14,15,17,19}$; movimentos oculares pela eletro-oculografia ${ }^{18}$ e qualidade de vida ${ }^{14}$. Três dos artigos descrevem aspectos relacionados a propriedades psicométricas da IM $\mathrm{IM}^{4,16,19}$.

De acordo com o nível de evidência avaliado, houve predomínio de ensaios clínicos randomizados (ERC), classificados como nível 1 de evidência, sendo esses estudos considerados de forte recomendação, além de um estudo transversal nível 3 de evidência ${ }^{16}$, e um relato de caso nível 5 de evidência ${ }^{20}$. O conhecimento da classificação de evidências proporciona subsídios para o fisioterapeuta na avaliação crítica de resultados oriundos de pesquisas e, consequentemente, na tomada de decisão sobre a incorporação das evidências à prática clínica ${ }^{21}$. 
Tabela 1. Síntese dos estudos de IM em neurorreabiliação, categorizados por ordem crescente do ano de publicação.

\begin{tabular}{|c|c|c|c|c|c|c|}
\hline $\begin{array}{c}\text { Autor e } \\
\text { Ano }\end{array}$ & $\begin{array}{l}\text { Tipo de } \\
\text { Estudo }\end{array}$ & Amostra & $\begin{array}{l}\text { Protocolo de } \\
\text { Intervenção }\end{array}$ & Medidas & Desfechos & Resultados \\
\hline $\begin{array}{l}\text { Heremans et } \\
\text { al. } 2011^{4}\end{array}$ & ERC & DP & $\begin{array}{l}\text { GE (n14) e GC (n14) } \\
\text { foram avaliados na } \\
\text { realização de testes } \\
\text { específicos sobre a } \\
\text { capacidade } \\
\text { geração de imagens } \\
\text { motoras. }\end{array}$ & $\begin{array}{c}\text { H\&Y } \\
\text { MEEM } \\
\text { UPDRS } \\
\text { SCOPA-COG } \\
\text { MIQ-R } \\
\text { KVIQ } \\
\text { CMIA } \\
\text { EVA } \\
\text { Teste de } \\
\text { Caixa de } \\
\text { Blocos }\end{array}$ & $\begin{array}{l}\text { Capacidade de } \\
\text { Imaginar. }\end{array}$ & 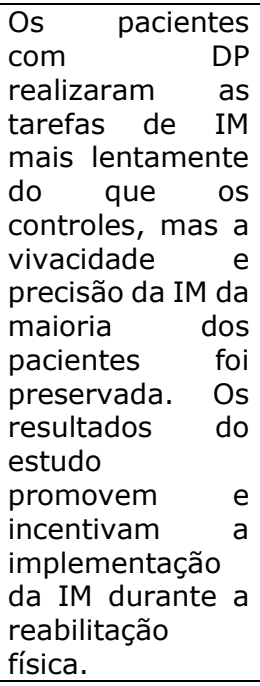 \\
\hline $\begin{array}{l}\text { Braun et al. } \\
2008^{1}\end{array}$ & $\begin{array}{c}\text { ERC } \\
\text { Multicêntrico }\end{array}$ & $\mathrm{DP}$ & $\begin{array}{l}\text { GE (n25)=PF e PM; GC } \\
\text { (n22)=PF } \\
\text { Relaxamento; } 1 \mathrm{~h} \text { por } \\
\text { semana, ou } 2 \text { sessões } \\
\text { de } 30 \text { minutos, } \\
\text { durante } 6 \text { sem., com } \\
\text { follow-up de } 3 \text { meses. }\end{array}$ & $\begin{array}{l}\text { MEEM } \\
\text { H\&Y } \\
\text { EVA } \\
\text { TUG } \\
\text { TC10m }\end{array}$ & $\begin{array}{c}\text { Mobilidade } \\
\text { funcional - } \\
\text { Marcha; } \\
\text { Marcha } \\
\text { comunitária. }\end{array}$ & $\begin{array}{l}\text { Neste estudo não } \\
\text { houve melhora } \\
\text { no GE } \\
\text { comparado ao } \\
\text { tratamento } \\
\text { oferecido neste } \\
\text { estudo ao GC. A } \\
\text { PM não ofereceu } \\
\text { benefício } \\
\text { adicional a PF. }\end{array}$ \\
\hline $\begin{array}{l}\text { Letswaart et } \\
\text { al. } 2011^{12}\end{array}$ & ERC & $\begin{array}{c}\text { AVC } \\
\text { (Subagudo) }\end{array}$ & $\begin{array}{l}\text { GE } 1(n=39): \text { PM e GE } \\
2(n=31): \text { PM não } \\
\text { motora com a mesma } \\
\text { intensidade. PM } 45 \\
\text { min, 3x na sem, por } 4 \\
\text { sem. e sessões } \\
\text { independentes } 2 x \text { na } \\
\text { sem, por 30min, sob a } \\
\text { perspectiva de } 1 \text { a } \\
\text { pessoa para o GE1. } \\
\text { GC }(n=32): \text { PF sem } \\
\text { treinamento adicional. } \\
\text { PM } 45 \text { min/3x na sem, } \\
\text { por } 4 \text { sem e sessões } \\
\text { independentes de } \\
30 \text { min, } 2 x \text { na sem }\end{array}$ & $\begin{array}{c}\text { ARAT } \\
\text { Força de } \\
\text { Preensão } \\
\text { IB } \\
\text { Perfil de } \\
\text { Limitação } \\
\text { Funcional }\end{array}$ & $\begin{array}{c}\text { Funcionalidade } \\
\text { MS. }\end{array}$ & $\begin{array}{l}\text { A PM não } \\
\text { favorece a } \\
\text { recuperação } \\
\text { motora logo após } \\
\text { o AVC. Será a PM } \\
\text { uma técnica de } \\
\text { reabilitação } \\
\text { válida por si só? }\end{array}$ \\
\hline $\begin{array}{l}\text { Grangeon et } \\
\text { al. } 2012^{20}\end{array}$ & $\begin{array}{l}\text { Estudo de } \\
\text { Caso }\end{array}$ & LM (C6) & 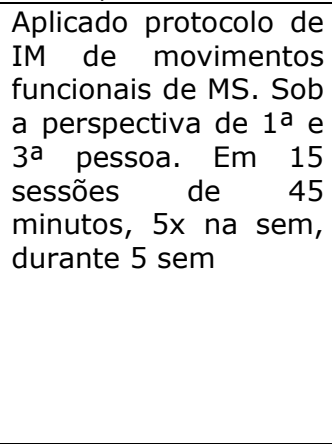 & $\begin{array}{c}\text { KVIQ } \\
\text { Cronometria } \\
\text { Mental } \\
\text { Análise } \\
\text { Eletrodérmica } \\
\text { Avaliação de } \\
\text { Minnesota } \\
\text { Teste de } \\
\text { Caixa de } \\
\text { Blocos } \\
\text { Medidas } \\
\text { Cinemáticas }\end{array}$ & $\begin{array}{l}\text { Desempenho } \\
\text { motor MS. }\end{array}$ & $\begin{array}{l}\text { Mobilidade } \\
\text { qualidade dos } \\
\text { movimentos de } \\
\text { MS melhorados, } \\
\text { após intervenção } \\
\text { com IM. Com } \\
\text { efeito percebido } \\
\text { até } 3 \text { meses após } \\
\text { treinamento. } \\
\text { Este estudo } \\
\text { apoia a } \\
\text { viabilidade da IM } \\
\text { na PF. }\end{array}$ \\
\hline
\end{tabular}


Tabela 1 (cont.). Síntese dos estudos de IM em neurorreabiliação, categorizados por ordem crescente do ano de publicação.

\begin{tabular}{|c|c|c|c|c|c|c|}
\hline Autor e Ano & $\begin{array}{l}\text { Tipo de } \\
\text { Estudo }\end{array}$ & Amostra & $\begin{array}{ll}\text { Protocolo } & \text { de } \\
\text { Intervenção } & \end{array}$ & Medidas & Desfechos & Resultados \\
\hline $\begin{array}{l}\text { Heremans et } \\
\text { al. } 2012^{18}\end{array}$ & ECR & EM & $\begin{array}{l}\text { GE (n14) e GC (n14)= } \\
\text { Tarefa funcional de IM } \\
\text { para MS e exercícios } \\
\text { funcionais, guiados } \\
\text { pelo uso de PE (pistas } \\
\text { auditivas e visuais). }\end{array}$ & $\begin{array}{c}\text { EDSS } \\
\text { MEEM } \\
\text { BTN } \\
\text { Teste dos } \\
\text { Nove Pinos } \\
\text { no Buracos. } \\
\text { KVIQ } \\
\text { Teste de } \\
\text { Rotação da } \\
\text { Mão } \\
\text { Teste de } \\
\text { Caixa de } \\
\text { Blocos }\end{array}$ & $\begin{array}{c}\text { Movimentos } \\
\text { horizontais } \\
\text { dos olhos pela } \\
\text { EOG; } \\
\text { EMG; } \\
\text { Cinemática de } \\
\text { pulso. }\end{array}$ & $\begin{array}{l}\text { Tanto o } \text { GE } \\
\text { sujeitos com EM, } \\
\text { como o GC } \\
\text { sujeitos } \\
\text { saudáveis, foram } \\
\text { afetados } \\
\text { positivamente } \\
\text { pelo uso da PE } \\
\text { para a realização } \\
\text { da IM. O estudo } \\
\text { aponta que PE } \\
\text { pode melhorar a } \\
\text { qualidade da } \\
\text { imaginação em } \\
\text { pacientes com } \\
\text { EM. }\end{array}$ \\
\hline $\begin{array}{l}\text { Dickstein et } \\
\text { al. } 2013^{15}\end{array}$ & $\begin{array}{c}\text { ECR } \\
\text { Crossover }\end{array}$ & $\begin{array}{c}\text { AVC } \\
\text { (Crônico) }\end{array}$ & $\begin{array}{l}\text { GE (n13)=IM de } \\
\text { locomoção em casa e } \\
\text { comunitária, sob a } \\
\text { perspectiva de } 1^{a} \text { e } 3^{a} \\
\text { pessoa; GC (n12) = } \\
\text { PF de exercícios } \\
\text { funcionais de MS. Foi } \\
\text { incluido componente } \\
\text { motivacional no } \\
\text { protocolo. } \\
\text { duração total de } 8 \\
\text { semanas, 3x na sem, } \\
\text { com follow-up de } \\
\text { 2sem. }\end{array}$ & $\begin{array}{l}\text { TC10m. } \\
\text { Escala de } \\
\text { Tinetti } \\
\text { FES } \\
\text { SAM }\end{array}$ & $\begin{array}{c}\text { Marcha } \\
\text { Domiciliar; } \\
\text { Marcha } \\
\text { Comunitária. }\end{array}$ & $\begin{array}{l}\text { A marcha } \\
\text { domiciliar } \\
\text { melhorou após } \\
\text { aplicação do } \\
\text { protocolo } \\
\text { enquanto a } \\
\text { marcha } \\
\text { comunitária não. } \\
\text { Efeito positivo na } \\
\text { autoeficácia } \\
\text { relacionada a } \\
\text { quedas, também } \\
\text { foi observado. } \\
\text { Além disso, as } \\
\text { mudanças } \\
\text { permaneceram } \\
\text { observáveis por } \\
\text { mais duas } \\
\text { semanas. }\end{array}$ \\
\hline $\begin{array}{l}\text { Myers et al. } \\
2018^{5}\end{array}$ & ECR & DP & $\begin{array}{l}37 \text { participantes, } 13 \\
\text { sujeitos com e } 24 \text { sem } \\
\text { congelamento. } \\
\text { Durante } 12 \text { semanas, } \\
\text { praticaram IM e } \\
\text { execução das tarefas: } \\
\text { caminhada }(n=11) \text {, } \\
\text { dança - tango }(n=11) \\
\text { e alongamento guiado } \\
(n=13) \text {. }\end{array}$ & $\begin{array}{l}\text { H\&Y } \\
\text { MEEM; } \\
\text { UPDRS; } \\
\text { RMF } \\
\text { FOG-Q } \\
\text { KVIQ }\end{array}$ & $\begin{array}{l}\text { Sinal RMF; } \\
\text { Marcha. }\end{array}$ & $\begin{array}{l}\text { As evidências } \\
\text { sugerem possível } \\
\text { redução } \\
\text { progressiva na } \\
\text { atividade } \\
\text { regiões de } \\
\text { associadas a } \\
\text { região } \\
\text { somatossensorial } \\
\text { nos pacientes } \\
\text { com } \\
\text { congelamento. } \\
\text { No entanto, um } \\
\text { programa de } 12 \\
\text { semanas não foi } \\
\text { capaz de gerar } \\
\text { mudanças } \\
\text { mensuráveis na } \\
\text { marcha. }\end{array}$ \\
\hline $\begin{array}{l}\text { Nascimento } \\
\text { et al. } 2019^{17}\end{array}$ & ERC & DP & $\begin{array}{l}\text { GE (n20) = IM marcha } \\
\text { sob a perspectiva de } \\
\text { 3a pessoa; GC (n20) } \\
=\text { PF marcha. Em } 12 \\
\text { sessões de } 90 \\
\text { minutos, 3x na sem, } \\
\text { por } 4 \text { sem. }\end{array}$ & $\begin{array}{c}\text { Análise } \\
\text { Cinemática } \\
\text { da Marcha } \\
\text { EEG } \\
\text { TUG }\end{array}$ & $\begin{array}{c}\text { Mobilidade } \\
\text { Funcional - } \\
\text { Marcha. }\end{array}$ & $\begin{array}{l}\text { Melhora da } \\
\text { marcha, } \\
\text { mobilidade e da } \\
\text { atividade } \\
\text { eletromiográfica } \\
\text { nos sujeitos com } \\
\text { DP. A IM em um } \\
\text { programa de } \\
\text { reabilitação se } \\
\text { mostra efetiva, } \\
\text { de fácil } \\
\text { aplicabilidade e } \\
\text { baixo custo. }\end{array}$ \\
\hline
\end{tabular}


Tabela 1 (cont.). Síntese dos estudos de IM em neurorreabiliação, categorizados por ordem crescente do ano de publicação.

\begin{tabular}{|c|c|c|c|c|c|c|}
\hline Autor e Ano & $\begin{array}{l}\text { Tipo de } \\
\text { Estudo }\end{array}$ & Amostra & $\begin{array}{ll}\text { Protocolo } & \text { de } \\
\text { Intervenção } & \end{array}$ & Medidas & Desfechos & Resultados \\
\hline $\begin{array}{l}\text { Guerra, et al. } \\
2008^{14}\end{array}$ & ERC & $\begin{array}{c}\text { AVC } \\
\text { (Subagudo) }\end{array}$ & $\begin{array}{l}\text { GE }(n 7)=P F \text { e PM; GC } \\
\text { (n7)=PF } \\
\text { Treinamento } \\
\text { Cognitivo. Durante } 12 \\
\text { sessões, em } \\
\text { semanas. }\end{array}$ & $\begin{array}{c}\text { MEEM } \\
\text { KVIQ-10 } \\
\text { FMA } \\
\text { TUG-ABS } \\
\text { TUG } \\
5 \text { mWT } \\
\text { DASS21 } \\
\text { WHOQOL }\end{array}$ & $\begin{array}{l}\text { Mobilidade } \\
\text { Funcional - } \\
\text { Marcha; } \\
\text { Qualidade de } \\
\text { Vida. }\end{array}$ & $\begin{array}{l}\text { Os efeitos da } \\
\text { associação entre } \\
\text { a PM e PF em } \\
\text { sujeitos com AVC } \\
\text { subagudo foram } \\
\text { evidenciados. }\end{array}$ \\
\hline $\begin{array}{l}\text { Morioka et } \\
\text { al. } 2019^{16}\end{array}$ & $\begin{array}{l}\text { Transversal } \\
\text { Multicêntrico }\end{array}$ & $\begin{array}{c}\text { AVC } \\
\text { (Agudo e } \\
\text { Subagudo) }\end{array}$ & $\begin{array}{l}31 \text { participantes com } \\
\text { AVC apresentando } \\
\text { lesão tanto no } \\
\text { hemisfério direito } \\
\text { como no esquerdo. }\end{array}$ & $\begin{array}{l}\text { OI } \\
\text { BCT } \\
\text { FMA } \\
\text { AOU } \\
\text { QOM } \\
\text { MAL }\end{array}$ & $\begin{array}{c}\text { Capacidade de } \\
\text { Imaginar; } \\
\text { Função MS } \\
\text { parético. }\end{array}$ & $\begin{array}{l}\text { Em pacientes } \\
\text { com AVC a OI de } \\
\text { imagem afeta } \\
\text { diretamente a } \\
\text { quantidade e } \\
\text { qualidade do } \\
\text { movimento do } \\
\text { MS afetado, } \\
\text { influenciando } \\
\text { diretamente as } \\
\text { AVDS. }\end{array}$ \\
\hline $\begin{array}{l}\text { Paddo et al. } \\
2020^{19}\end{array}$ & ERC & EM & $\begin{array}{l}\text { GE (n15) e GC (n15) } \\
\text { foram convidados a } \\
\text { percorrerem } 3 \\
\text { caminhos de mesmo } \\
\text { comprimento, porém } \\
\text { larguras distintas. Isto } \\
\text { de forma real e após } \\
\text { mentalmente, sob a } \\
\text { perspectiva de } 1^{a} \\
\text { pessoa, }\end{array}$ & $\begin{array}{c}\text { EDSS } \\
\text { SDMT } \\
\text { T25-FW } \\
\text { IPAQ } \\
\text { DTQ } \\
\text { KVIQ } \\
\text { MFIS }\end{array}$ & $\begin{array}{c}\text { Anisocronia } \\
\text { entre a } \\
\text { locomoção } \\
\text { real e mental. }\end{array}$ & 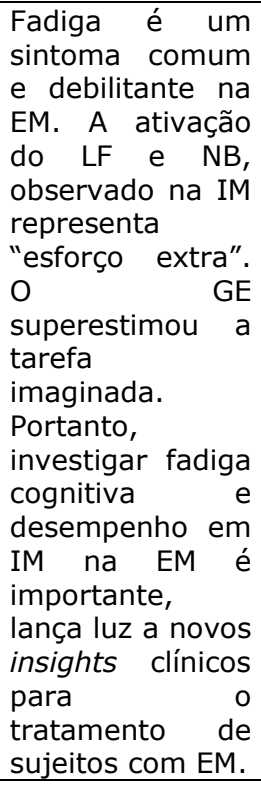 \\
\hline
\end{tabular}

Ensaio Clinico Randomizado (ERC); Doença de Parkinson (DP); Grupo Experimental (GE), Grupo Controle (GC); Escala de Estadiamento de Hoehn \& Yahr (H\&Y); Miniexame do Estado Mental (MEEM); Escala Unificada de Avaliação para Doença de Parkinson (UPDRS); Escala de Desfechos da Doença de Parkinson (SCOPA-COG); Questionário de Imagética Motora (MIQ);Questionário de Imagética Motora Visual e Cinestésico (KVIQ-10); Avaliação de Imagens Motoras Caóticas (CMIA); Escala Visual Analógica (EVA); Imagética Motora (IM); Prática Física (PF); Teste de Caminhar e Levantar Cronometrado (TUG); Teste de Caminhada 10m (TC10m); Acidente Vascular Cerebral (AVC); Prática Mental (PM); Teste de Ação de Extremidade Superior (ARAT);Índice de Barthel (IB); Perfil de Limitação Funcional (FLP); Membro Superior (MS); Lesão Medular (LM);Esclerose Múltipla (EM); Escala de Incapacidade Funcional Expandida (EDSS); Bateria de Testes Neuropsicológicos (BTN); Eletro-Oculografia (EOG); Eletromiografia (EMG); Pistas Externas (PE); Escala de Eficácia de Quedas (FES); Monitor de atividade StepWatch (SAM); Monitor de Atividade StepWatch (SAM); Ressonância Magnética Funcional (RMF); Questionário de Congelamento da Marcha (FOG-Q); Eletroencefalografia (EEG); Avaliação de Fugl-Meyer (FMA); Avaliação de Estratégias Biomecânicas (TUG-ABS); Teste de Velocidade da Marcha em 5m (5mWT); Escala de Depressão, Ansiedade e Estresse (DASS-21); Avaliação da Qualidade de Vida da Organização Mundial da Saúde (WHOQOL); Índice de Ovalização (OI); Tarefa de Acoplamento Bimanual (BCT); Quantidade de Uso (AOU); Qualidade do Movimento (MAL); Atividade Básica de Vida Diária (AVDS); Teste de Modalidades de Simbolos e Dígitos (SDMT); Teste de Caminhada Cronometrado dos 25 pés (T25-FW); Questionário Internacional de Atividade Física (IPAQ);Questionário de Dupla Tarefa (DTQ); Escala Modificada do Impacto da Fadiga (MFIS); Lobo Frontal (LF); Núcleo da Base (NB). 


\section{DISCUSSÃO}

\section{Definindo Imagética Motora.}

A neurociência cognitiva tem discutido o movimento imaginado sob diferentes termos, incluindo imagética motora, imagens mentais, imagens de movimento, prática mental, ensaio de imagens, simulação mental, visualização, imagens cinestésicas e ensaio comportamental visuomotor $^{22}$. Não surpreendentemente, o uso desses termos é sustentado por vários conceitos ligeiramente diferentes de "movimento imaginário do corpo". Nesta revisão, descreveremos os termos Imagética Motora (IM) e Prática Mental (PM), e estes também serão utilizados como sinônimos.

A IM é definida como a execução mental de um movimento sem qualquer movimento evidente ou ativação muscular 23,24 . A IM representa o resultado do acesso consciente à intenção de um movimento, que geralmente é realizado inconscientemente durante a preparação motora, estabelecendo uma relação entre eventos motores a percepções cognitivas ${ }^{25}$. Essa habilidade de imaginar um movimento, tanto de forma implícita ou explicita tem sido usada para aprender habilidades motoras ${ }^{24}$.

Há evidências de que circuitos neurais que envolvem o córtex motor primário, o cerebelo e os núcleos da base são ativados durante a imaginação do movimento. Além disso, a teoria da programação motora sugere que um dos componentes fundamentais para que um movimento eficaz ocorra é a capacidade de realizar representações mentais 
desse movimento. Isso ocorre porque essas representações servem como um modelo interno para facilitar os processos de planejamento da ação ${ }^{24}$.

São reconhecidas duas abordagens possíveis para a IM: IM interna e externa24,25. A IM interna tem caráter cinestésico, o sujeito realiza uma simulação mental tentando sentir o movimento, sem que isso de fato ocorra. Usando a perspectiva interna o movimento é imaginado a partir de uma visão em primeira pessoa, por exemplo, como se estivesse vendo com os próprios olhos a parte do corpo que se move ${ }^{22}$. Já a IM externa, é predominantemente visual, sendo uma visão na perspectiva de terceira pessoa, semelhante a um observador assistindo à execução de um movimento na televisão ${ }^{22}$. As imagens visuais também podem ser divididas em imagens visuais de primeira pessoa (uma perspectiva subjetiva da própria pessoa) e imagens visuais de terceira pessoa (uma perspectiva objetiva como uma terceira pessoa $)^{23,26}$.

Estudos de neuroimagem demonstram ser a atividade cerebral diferente entre IM cinestésica e a IM visual ${ }^{23}$. Em estudo utilizando RMF foi comparada a atividade cerebral entre IM cinestésica e IM visual ${ }^{27}$. As imagens cinestésicas produziram maior atividade nas regiões da área motora suplementar, córtex pré-motor, cerebelo e lóbulo parietal inferior. Em contraste, a IM visual mostrou maior ativação no córtex occipital e lobos parietais superiores. Esses resultados, sugerem que as estratégias de IM são mediadas por sistemas neurais distintos, que contribuem de forma 
diferenciada durante os processos de aprendizagem e reabilitação neurológica.

A Prática Mental tem sido reconhecida como, um ensaio mental voluntário de um gesto ou tarefa motora ${ }^{28}$. É um método pelo qual a reprodução interna de um determinado ato motor é repetida extensivamente com a intenção de melhora da performance. As primeiras concepções entenderam a PM como um processo abstrato e simbólico, como "o ensaio mental de uma atividade física na ausência de quaisquer movimentos musculares grosseiros". A PM é uma intervenção terapêutica promissora, pois quando associada a terapia física ou ocupacional pode apresentar ganhos funcionais ${ }^{29}$. Assim, PM é uma estratégia cognitiva que pode favorecer a aquisição de habilidades motoras e funcionais em indivíduos com lesões neurológicas ${ }^{3}$.

Embora a PM seja utilizada há tempos por diferentes disciplinas ${ }^{22}$, há pouco tempo começou a ser aplicada no contexto da reabilitação. Várias são as evidências que suportam seus benefícios na melhora do desempenho motor em sujeitos com disfunções neuromotoras ${ }^{5,24,25}$. Por ser um método de baixo custo e acessível, parece importante o conhecimento e divulgação ao fisioterapeuta sobre seu uso.

\section{Avaliação da capacidade de gerar imagens mentais}

Considerando que IM é um construto multidimensional22, medimos seus processos usando uma combinação de testes psicométricos ${ }^{30}$, procedimentos qualitativos ${ }^{30}$, métodos cronométricos, como o paradigma de 
cronometria mental, que tem sido usado para investigar a IM comparando a duração necessária para a execução da ação real e imaginária e técnicas psicofisiológicas ${ }^{30}$. Para 0 indivíduo se beneficiar da PM, ele deve ter certa capacidade de gerar imagens mentais, embora isso possa ser desenvolvido e melhorado. Essa habilidade tem características heterogêneas que diferem entre os indivíduos. Por se tratar de uma operação cognitiva complexa, acaba tornando a sua medição uma tarefa difícil26. O mais apropriado para obter uma melhor compreensão desta habilidade é avaliar os diferentes domínios da IM. Várias avaliações foram desenvolvidas nas últimas décadas a fim de avaliar a capacidade de gerar imagens mentais de um indivíduo dentro de diferentes dimensões, por exemplo, vivacidade, facilidade, precisão ou clareza da imagem. Algumas avaliações podem até avaliar ambas as dimensões.

Os métodos para avaliação da IM, podem ser divididos em:

- Abordagem psicométrica, estes envolvem questionários padronizados autoadministrados que avaliam a vivacidade das imagens mentais ${ }^{23}$. Os sujeitos são instruídos a gerarem uma imagem mental e a partir disso pontuarem em uma escala do tipo Likert, o quão vívida a imagem é. Os primeiros questionários construídos com esse objetivo foram: Vividness of Movement Imagery Questionnaire (VMIQ) (Klinkas, et al 2017) e Movement Imagery Questionnaire (MIQ) ${ }^{29}$. 
- Abordagem qualitativa, que tem sido utilizada através de entrevistas semiestruturadas ${ }^{26}$, onde o indivíduo narra retrospectivamente sua experiência de imaginação. Sua subjetividade o torna complementar as outras técnicas de avaliação.

- Medidas cronométricas, testes de resolução de problemas espaço temporais são aplicados. Estes ocorrem através da manipulação de imagens - teste de rotação mental23, e cronometria mental, que se define como a congruência temporal entre um ato motor executado e imaginado. Esses métodos são simples, confiáveis e avaliam as características temporais da precisão das imagens.

- Técnica funcionais e de neuroimagem, estes métodos avaliam as mudanças da atividade cerebral, são eles: a Ressonância Magnética Funcional (RMF), Tomografia por Emissão de Pósitrons (PET), Estimulação Magnética Transcraniana (EMT) e Eletroencefalografia (EEG) ${ }^{23}$.

- Medidas neurofisiológicas, são usadas para a avaliação da condutividade eletrodérmica, resposta cardiovascular e respiratório (frequência cardíaca e respiratória), avaliando dessa forma a variabilidade do sistema nervosos autônomo entre os indivíduos ${ }^{26}$.

Embora as avaliações da habilidade de IM sejam fáceis de usar e possuem um bom custo-benefício, essas possuem algumas limitações, como por exemplo, não permitem o controle da capacidade de IM. A literatura recomenda que esta habilidade é mais bem avaliada quando usado uma 
combinação das medidas qualitativa, psicométrica, cronométrica e abordagens psicofisiológicas ${ }^{22,30}$.

\section{Evidências do treinamento com IM em populações de pacientes}

Quando se trata da intervenção determinada pelo paradigma de IM no âmbito da neurorreabilitação a literatura aborda indivíduos com algumas condições de saúde. Dentre elas as mais comuns são: Acidente Vascular Cerebral (AVC), Doença de Parkinson (DP), Esclerose Múltipla (EM) e Lesão Medular $(L M)^{1,5,13,18}$. Apesar de alguns estudos demonstrarem benefícios dessa intervenção em desfechos como velocidade da marcha, função do membro superior, aprendizagem motora, tamanho do passo, otimização de transferências etc ${ }^{1,5,13,15}$, outras pesquisas sugerem que essa estratégia não determina efeitos palpáveis ${ }^{12}$. Dessa forma, os resultados acerca da abordagem permanecem heterogêneos. Assim como sua forma de implementação ${ }^{13}$. Nesta seção serão destacados algumas dessas análises.

A maior parte dos estudos que identificam efeitos de IM no âmbito da neurorreabilitação abordam sujeitos com sequelas de AVC. No estudo com o objetivo principal de testar os resultados de uma intervenção baseada em tal paradigma, o conteúdo motor foi integrado a estratégias motivacionais ${ }^{1}$. Como desfechos de interesse eles analisaram a marcha e a autoeficácia relacionada a quedas de indivíduos residentes na comunidade com hemiparesia crônica pós AVC. Para isso a amostra foi dividida em dois subgrupos sendo um 
experimental que recebia as intervenções propostas com imaginação de tarefas de deambulação e um controle que recebiam intervenções propostas com imaginação de tarefas que envolviam o membro superior (MS). Após análise foi verificado um aumento da velocidade de caminhada dos indivíduos que compuseram o grupo experimental quando comparado aos controles. Entretanto, não houve diferença significante entre os grupos quando verificados o número de passos dados por minuto durante a hora mais ativa do dia e o número de passos em um tempo pré-definido. Em relação a autoeficácia para quedas efeitos positivos foram verificados para os dois grupos, dessa forma, o resultado não pode ser atribuído a intervenção proposta. Além disso, os autores destacam que o nível de autoeficácia antes da abordagem já era elevado, sendo assim, o espaço para melhorias era limitado.

Para investigar se os efeitos da PM ocorrem devido a própria intervenção ou são decorrentes do incentivo e atenção adicional fornecidos pelo terapeuta, foi conduzido um ensaio randomizado cego e simples, contando com uma grande amostra de pacientes com sequelas de AVC com fraqueza persistente em $\mathrm{MS}^{12}$. Os indivíduos foram alocados em 3 grupos experimental, estes receberam o treino com imagens motoras e dois grupos controle, um denominado de grupo atenção controle, que realizava ensaio mental não relacionado ao controle motor e o outro denominado controle que recebia somente alguns cuidados sem treinamento adicional. Os desfechos de interesse foram o teste de ação 
das extremidades superiores (ARAT), o índice de Barthel e o perfil de limitação funcional. Após análise foi verificado que os resultados desse estudo não mostraram nenhuma evidência do benefício de PM com imagens motoras em pacientes com sequelas de AVC em qualquer medida de resultado.

Pesquisas com amostras compostas por indivíduos com DP também são controversas. Há resultados que determinam que imaginar uma ação seria possível caso houvesse adaptação do conteúdo as habilidades dos pacientes ${ }^{1}$. Outros destacam que a maioria dos pacientes com DP em estágio intermediário e inicial preservaram a vivacidade da IM e a precisão. Em contrapartida há relatos na literatura que esses indivíduos não são capazes de realizar imagens mentais ${ }^{18}$. O estudo de Braun et al $^{1}$ apresentou duas questões de pesquisa: 1- A PM associada a fisioterapia é mais efetiva na melhora da mobilidade de pessoas com DP na comunidade do que o relaxamento associado a fisioterapia? 2- O quanto a gravidade da doença influencia no efeito do tratamento desses sujeitos? Para respondê-las os participantes da pesquisa foram expostos a 6 semanas de intervenção. Um grupo foi exposto a fisioterapia associada a PM, sendo denominado de grupo experimental e o outro que realizou um programa de fisioterapia associada a um programa de relaxamento, foi denominado como grupo controle. O principal objetivo das intervenções eram melhorar as tarefas locomotoras, como caminhar, levantar-se de uma cadeira ou do chão. Os 
participantes foram avaliados em 3 momentos: préintervenção, pós-intervenção e no follow up de 3 meses. Como resultado os autores verificaram que não houve diferença entre as intervenções e que as duas intervenções apresentaram efeitos benéficos.

O tema Esclerose Múltipla e IM ainda é pouco explorado na literatura. Há relatos que indivíduos com EM podem ter dificuldades na simulação mental de ações e que vários aspectos podem afetar o desempenho ${ }^{18}$. Adicionalmente, foram encontrados prejuízos na precisão e organização temporal que poderiam limitar os benefícios da prática de $\mathrm{IM}^{18}$. Devido a isso, foi realizado um estudo com o objetivo de verificar se a qualidade da IM praticada por esses sujeitos pode ser otimizada por sinalizações externas (pistas visuais e auditivas $)^{18}$. Para isso os autores compararam 2 grupos, um com indivíduos saudáveis e outro com indivíduos com EM. Para implementar a análise eles utilizaram uma tarefa direcionada a um objetivo que envolvia o membro superior na presença e ausência de pistas. As variáveis tempo e precisão foram verificadas a partir de movimentos oculares. Já que estudos prévios encontraram uma correlação entre esses e os movimentos imaginados. O tempo e a amplitude do movimento ocular durante a imaginação e a execução do movimento foi maior em pacientes com EM quando comparado aos controles. Após a implementação das sinalizações externas houve redução do tempo e aumento da precisão espacial quando os indivíduos com EM foram expostos a pistas visuais nas duas condições de movimento. 
Dessa forma, o presente estudo mostra que pistas externas podem melhorar a qualidade da IM em pacientes com EM para um grau semelhante aos controles.

Estudos que reúnem os descritores Lesão Medular e IM também demonstram atenção limitada, apesar de algumas pesquisas que envolvem neuroimagem demonstrarem que a representação mental é mantida nesses indivíduos ${ }^{5}$. Numa pesquisa que aborda esses sujeitos, os autores conduziram um estudo de caso com o intuito de avaliar a viabilidade de um programa de reabilitação que combina IM e terapia convencional para melhora da função no MS em indivíduos

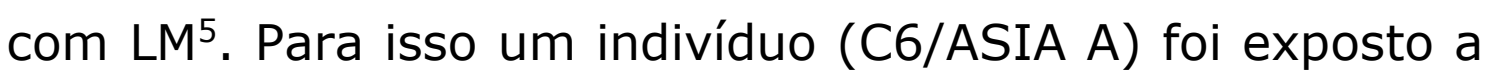
um treinamento de 15 sessões que tinha com o objetivo de melhorar o movimento da mão para alcance e preensão de objetos por tenodese. O sujeito foi avaliado antes e após as intervenções, além disso, um follow up de 3 meses foi conduzido após o término da abordagem. Como resultado verificou-se que o sujeito não apresentou dificuldade para imaginar a tarefa. Após intervenção o teste de Minnesota diminuiu em 1 minuto e 25 segundos e o teste de caixa de blocos melhorou em 3 unidades. A análise cinemática permitiu identificar melhora do tempo e da suavidade da trajetória do movimento. Os ganhos no desempenho permaneceram estáveis após 3 meses de participação. Dessa forma, entende-se que o programa de IM foi capaz de mudar a programação motora e foi capaz de reorganizar o transporte espacial da mão no paciente com LM. 


\section{CONCLUSÃO}

Há evidências do uso da PM na melhora de habilidades motoras em sujeitos com diferentes condições neurológicas. Nesta revisão, não foi observado consenso nos protocolos de intervenção. Observou-se a utilização de ambas as modalidades da técnica ( $1^{a}$ e $3^{a}$ pessoa), em relação a dosimetria, foi visto uma frequência de 2 a 5 vezes - em 4 a 8 semanas, com duração média de 55 minutos para as intervenções com PM e PF associados. Cronometria mental, abordagem psicométrica, respostas eletrodérmicas e cardiorrespiratórias, foram algumas das estratégias observadas para a avaliação da capacidade de gerar imagens e para monitorar o engajamento dos sujeitos.

Embora os estudos sejam promissores, ainda são controversos e limitados, existe uma grande variação na seleção de tarefas, de grupos, método de treinamento e uma série de outros fatores que podem explicar os diferentes resultados. No entanto, é uma técnica segura, facilmente aplicável e indicada como ferramenta adicional na reabilitação de indivíduos com disfunções neuromotoras.

\section{REFERÊNCIAS}

1.Braun SM, Kleynen M, Schols J, Beurskens A, Wade D. Using mental practice in stroke rehabilitation: a framework. Clin Rehabil 2008;22:579-91. https://doi.org/10.1177/0269215508090066

2.Kim SS, Lee $\mathrm{BH}$. Motor imagery training improves upper extremity performance in stroke patients. J Phys Ther Sci 2015;27:2289-91. https://doi.org/10.1589/jpts.27.2289

3.Timmermans AAA, Verbunt JA, Woerden RV, Moennekens M, Pernot $\mathrm{DH}$, Seelen HAM. Effect of Mental Practice on the Improvement of Function and Daily Activity Performance of the Upper Extremity in 
Patients With Subacute Stroke: A Randomized Clinical Trial. JAMDA 2013;14:204-12. https://doi.org/10.1016/j.jamda.2012.10.010

4. Heremans E, Feys P, Nieuwboer A, Vercruysse S, Vandenberghe W, Sharma N, et al. Motor Imagery Ability in Patients With Early- and MidStage Parkinson Disease. Neurorehabil Neural Repair 2011;25:168-77. https://doi.org/10.1177/1545968310370750

5. Myers OS, Mcneely ME, Pickett KA, Duncan RP, Earhart GM. Effects of exercise on gait and motor imagery in people with Parkinson disease and freezing of gait. Parkinsonism Relat Dis 2018;53:89-95. https://doi.org/10.1016/j.parkreldis.2018.05.006

6. Hanson M, Concialdi M. Motor imagery in multiple sclerosis: exploring applications in therapeutic treatment. J Neurophysiol 2019;1121:3479. https://doi.org/10.1152/jn.00291.2018

7.Thomschewski A, Ströhlein A, Langthaler PB, Schmid E, Potthoff J, Höller $\mathrm{P}$, et al. Imagine There Is No Plegia. Mental Motor Imagery Difficulties in Patients with Traumatic Spinal Cord Injury. Front Neurosci 2017;11:689. https://doi.org/10.3389/fnins.2017.00689

8.Liu K, Chan C, Lee TM, Hui-Chan CW. Mental imagery for promoting relearning for people after stroke: a randomized controlled trial. Arch Phys Med Rehabil 2004;85:1403-8. https://doi.org/10.1016/j.apmr.2003.12.035

9.Decety J. The neurophysiological basis of motor imagery. Behav Brain Res 1996;77:45-52. https://doi.org/10.1016/01664328(95)00225-1

10.Malouin F, Richards CL, Jackson PL, Dumas F, Doyon J. Brain activations during motor imagery of locomotor-related tasks: a PET study. Hum Brain Mapp 2003;19:47-62. https://doi.org/10.1002/hbm.10103

11. Malouin F, Richards RC. Mental Practice for Relearning Locomotor Skills. Phys 2010;90:240-51. https://doi.org/10.2522/ptj.20090029

12.Letswaart $M$, Johnston $M$, Dijkerman $H C$, Joice $S$, Scott $C L$, MacWalter RS, et al. Mental practice with motor imagery in stroke recovery: randomized controlled trial of efficacy. Brain 2011;134:1373-86. https://doi.org/10.1093/brain/awr090

13.Carrasco DG, Cantalapiedra AJ. Efectividad de la imaginería o práctica mental em la recuperación funcional tras el ictus: revisión sistemática. Neurología 2016;31:43-52. https://doi.org/10.1016/j.nrl.2013.02.003

14.Guerra ZF, Bellose LC, Faria CDCM, Lucchetti G. The effects of mental practice based on motor imagery for mobility recovery after subacute stroke: Protocol for a randomized controlled trial. Complem Ther Clin Pract 2008;33:36-42. https://doi.org/10.1016/j.ctcp.2018.08.002

15.Dickstein R, Deutsch JE, Yoeli Y, Kafri M, Falash F, Dunsky A, et al. Effects of Integrated Motor Imagery Practice on Gait of Individuals With Chronic Stroke: A Half-Crossover Randomized Study. Arc Phys Med Rehabil 2013;94:2119-25. https://doi.org/10.1016/j.apmr.2013.06.031 
16. Morioka S, Osumi M, Nishi $Y$, Ishigaki T, Ishibashi R, Sakauchi T, et al. Motor-imagery ability and function of hemiplegic Upper limb in stroke patients. Neurology 2019;6:596604. https://doi.org/10.1002/acn3.739

17. Nascimento IAPA, Santiago LMM, Souza AA, Pegado CL, Ribeiro TS, Rodrigues AR. Effects of motor imagery training of Parkinson's disease: a protocol for a randomized clinical trial. Trials 2019;20:626. https://doi.org/10.1186/s13063-019-3694-8

18. Heremans E, Nieuwboer A, Spildooren J, Bonddt S, D'Hooge AM, Helsen W, et al. Cued motor imagery in patients with multiple sclerosis. Neuroscience 2012;206:115-21. https://doi.org/10.1016/j.neuroscience.2011.12.060

19.Paddo J, Pedullà L, Bragadin MM, Piccardo E, Battaglia MA, Brichetto $\mathrm{G}$, et al. Spatial constraints and cognitive fatigue affect motor imagery of walking in people with multiple sclerosis. Nature 2020;14. https://doi.org/10.1038/s41598-020-79095-3

20.Grangeon M, Revol P, Guillot A, Rode G, Collet C. Could motor imagery be effective in upper limb rehabilitation of individuals with spinal cord injury? A case study. Spinal Cord 2012;50:766-71. https://doi.org/10.1038/sc.2012.41

21. Melnyk BM, Fineout-Overholt E, Stillwell SB, Williamson KM. Evidence-Based Practice: Step by Step: The Seven Steps of Evidence-Based Practice. Am J Nurs 2010;110:51-3. https://doi.org/10.1097/01.NAJ.0000366056.06605.d2

22.Suica Z, Platteau-Waldmeier P, Koppel S. Motor imagery ability assessments in four disciplines: protocol for a systematic review. BMJ Open 2018;8:e023439. http://dx.doi.org/10.1136/bmjopen-2018$\underline{023439}$

23. Mizuguchi $\mathrm{N}$, Nakata $\mathrm{H}$, Uchida $\mathrm{Y}$, Kanosue $\mathrm{K}$. Motor imagery and sport performance. J Phys Fitness Sports Med 2012;1:103-11. https://doi.org/10.7600/jpfsm.1.103

24.López ND, Pereira EM, Centeno EJ, Page JCM. Motor imagery as a complementary technique for functional recovery after stroke: a systematic review. Top Stroke Rehabil 2019;6:576-87. https://doi.org/10.1080/10749357.2019.1640000

25. Machado TC, Carregosa AA, Santos MS, Ribeiro NMS, Melo A. Efficacy of motor imagery additional to motor based therapy in the recovery of motor function of the upper limb in post-stroke individuals: a systematic review. Top Stroke Rehabil 2019;26:1-6. https://doi.org/10.1080/10749357.2019.1627716

26. Klinkas MM, Nagy SN, Ubillos-Landa S. Revisión sistemática sobre instrumentos de valoración de la imaginación motora para población hispanohablante: su uso en rehabilitación. Rev Neurol 2017;65:38595. https://doi.org/10.33588/rn.6509.2017084

27. Guillot A, Collet C, Nguyen VA, Malouin F, Richards C, Doyon J. Brain activity during visual versus kinesthetic imagery: an fMRI study. Hum Brain Map 2009;30:2157-72. https://doi.org/10.1002/hbm.20658 28. Braun S, Kleynen M, Theel TV, Kruithof N, Wade D, Beurskens A. The effects of mental practice in neurological rehabilitation; a 
systematic review and meta-analysis. Front Hum Neurosci 2013;2:390. https://doi.org/10.3389/fnhum.2013.00390

29.Butler AJ, Cazeaux J, Fidler A, Jansen J, Lefkove N, Gregg M, et al. The Movement Imagery Questionnaire-Revised, Second Edition (MIQRS) Is a Reliable and Valid Tool for Evaluating Motor Imagery in Stroke Populations. Evidence-Based Compl Alter Med 2012:1-11. https://doi.org/10.1155/2012/497289

30. Collet C, Guillot A, Lebon F, Macintyre T, Moran A. Measuring motor imagery using psychometric, behavioral, and psychophysiological tools. Exerc Sport Sci Rev 2011;39:85-92. 